\title{
Validación de la estructura interna del cuestionario de Satisfacción Laboral de Warr, Cox y Wall, mediante el método de estimación de Mínimos Cuadrados de Libre Escala
}

\author{
Víctor Ucedo Silva ${ }^{1}$ y Emma Cambillo Moyand ${ }^{2}$
}

Resumen: La investigación tuvo por objetivo evaluar la validez de la estructura interna y la fiabilidad del cuestionario de "Satisfacción Laboral de Warr, Cox y Wall" para ser aplicado a docentes de educación superior. Método: Mediante el Análisis Factorial Confirmatorio con el método de estimación de Mínimos Cuadrados de Libre Escala en modelos de medida unifactorial y bifactorial, en una muestra de 242 docentes de educación superior. Resultados: Obteniendo los indicadores Chi de 59.024 y 63.185, GFI de 0.984 y 0.983 , AGFI de 0.978 y 0.977 , RMSR de 0.066 y 0.070 , respectivamente, encontrándose dentro de los parámetros adecuados. El grado de fiabilidad se evaluó con el coeficiente Alpha de Cronbach=0.867 con un IC $(99 \%)[0.832 ; 0.897]$, el coeficiente de confiabilidad compuesto en el modelo unifactorial $\omega=0.908$ y en el bifactorial $\omega=0.913$, siendo la consistencia interna de la escala muy buena. Concluyendo que la estructura interna de la escala de satisfacción laboral es valida y es fiable.

Palabras clave: Validez, fiabilidad, satisfacción laboral, análisis factorial confirmatorio.

\section{Validation of the internal structure of the Warr, Cox and Wall Job Satisfaction questionnaire using the Free Scale Least Squares estimation method}

\begin{abstract}
The objective of the research was to evaluate the validity of the internal structure and the reliability of the "Warr, Cox and Wall Job Satisfaction" questionnaire to be applied to higher education teachers. Method: Using of the Confirmatory Factor Analysis with the method of estimation of Least Squares of Free Scale in unifactorial and bifactorial measurement models, in a sample of 242 higher education teachers. Results: Obtaining the indicators Chi of 59.024 and 63.185, GFI of 0.984 and 0.983 , AGFI of 0.978 and 0.977 , RMSR of 0.066 and 0.070 , respectively, being within the appropriate parameters. The degree of reliability was evaluated with Cronbach's Alpha coefficient $=0.867$ with a $\mathrm{CI}(99 \%)[0.832 ; 0.897]$, the composite reliability coefficient in the unifactorial model $\omega=0.908$ and in the bifactorial model $\omega=0.913$, being the internal consistency of the scale very better. Concluding that the internal structure of the job satisfaction scale is valid and reliable.
\end{abstract}

Keywords:Validity,reliability,job satisfaction,confirmatory factor analysis.

Recibido: 17/12/2020. Aceptado: 16/04/2021. Publicado online: 30/06/2021.

\footnotetext{
(c) Los autores. Este artículo es publicado por la Revista PESQUIMAT de la Facultad de Ciencias Matemáticas, Universidad Nacional Mayor de San Marcos. Este es un artículo de acceso abierto, distribuido bajo los términos de la licencia Creative Commons Atribucion-No Comercia-CompartirIgual 4.0 Internacional.(http://creativecommons.org/licenses/by-nc-sa/4.0/) que permite el uso no comercial, distribución y reproducción en cualquier medio, siempre que la obra original sea debidamente citada. Para uso comercial, por favor póngase en contacto con revistapesquimat.matematica@unmsm.edu.pe

${ }^{1}$ UNMSM, Facultad de Ciencias Matemáticas. e-mail: vucedos@unmsm.edu.pe

${ }^{2}$ UNMSM, Facultad de Ciencias Matemáticas, e-mail:ecambillom@unmsm.edu.pe
} 


\section{Introducción}

$\mathrm{Al}$ abordar el tema de la satisfacción laboral encontramos a lo largo del tiempo muchas investigaciones al respecto, esto puede deberse a sus posibles consecuencias que se presentarán al ser descuidadas ya que podrían presentarse riesgos psicosociales como el estrés, el cual es explicado en gran parte, por diferentes factores (somnolencia, fatiga, y caer en la rutina, es decir en una constante denominada "monotonía"), que a mediano o largo plazo conducen a enfermedades como la ansiedad, depresión y reacciones psicosomáticas. Por lo tanto, la satisfacción laboral está íntimamente relacionada con la salud de las personas, por tal motivo, la principal preocupación en la presente investigación es contar con un instrumento que mida la satisfacción laboral en los profesionales dedicados a la docencia en educación superior.

Contar con un instrumento que mida de manera adecuada la satisfacción laboral es una labor compleja, es necesario abordar esta problemática formulando las siguientes preguntas: ¿Existe una escala que nos permita medir la satisfacción laboral?, en especial ¿Esta o estas escalas de medición, tienen vigencia en la actualidad? y además ¿estas escalas han sido validadas para medir la satisfacción laboral de los docentes en las instituciones de educación superior? respecto al ¿Lugar? ¿Tiempo?.

Considerando las preguntas anteriores se ha elegido la escala de satisfacción laboral de Warr, Cox y Wall, el cual tiene una estructura bifactorial, pues propone medir la satisfacción intrínseca y extrínseca del individuo. Esta escala ha sido presentada en el artículo "Overall Job Satisfaction"por Warr, Cox y Wall en 1979, citado por Pérez y Fidalgo [17] y fue desarrollada para evaluar la satisfacción en el ámbito laboral. Actualmente no se cuenta con un instrumento validado para medir la satisfacción laboral en docentes de educación superior en el Perú, entonces es necesario formular la siguiente pregunta ¿El cuestionario de Satisfacción Laboral de Warr, Cox y Wall es válido en su estructura interna y es fiable para medir la satisfacción en docentes de educación superior en el Perú?.

\section{Métodos}

La población de estudio estuvo conformada por toda la plana docente de una corporación de educación superior del Perú, se consideró como criterio de inclusión que el docente tenga vínculo laboral con la corporación (con contrato laboral) se excluyeron a los docentes invitados, docentes reemplazantes y docentes de intercambio, el tamaño de muestra estuvo conformado por 242 docentes seleccionados al azar, mediante el muestreo aleatorio simple con un nivel de significación del $1 \%$.

\subsection{Instrumento}

El instrumento es la escala de satisfacción laboral propuesto por Warr, Cox y Wall (1979). El instrumento está conformado por 15 ítems, referidos al entorno laboral físico, la libertad para elegir el método de trabajo, colegas, entre otros. 


\section{Análisis Factorial}

Albright [10, p.1] afirma que: El Análisis Factorial es un método estadístico común que se utiliza para encontrar un pequeño conjunto de variables no observadas (también llamadas variables latentes o factores) que pueden explicar la covarianza entre un conjunto mayor de variables observadas (también llamadas variables explicativas).

El Análisis Factorial es una técnica multivariada que se divide en dos partes el análisis factorial exploratorio (AFE) y el análisis factorial confirmatorio (AFC).

Jöreskog et al. [10, p.283] afirman que: El análisis factorial exploratorio se utiliza cuando se tiene un conjunto de variables y se desea averiguar cuántos factores se necesitan para explicar sus intercorrelaciones y lo qué están midiendo estos factores. Tanto el número de factores como el significado de los factores son desconocidos. La interpretación y la denominación de los factores se hacen generalmente después de la rotación analítica.

Brown [6, pp. 18-19] indica que: El objetivo primordial de la AFE es evaluar la dimensionalidad de un conjunto de indicadores múltiples (por ejemplo, elementos de un cuestionario) descubriendo el menor número de factores interpretables necesarios para explicar las correlaciones entre ellos. El AFE es un análisis exploratorio"porque no se colocan restricciones a priori en el patrón de relaciones entre las medidas observadas y las variables latentes.

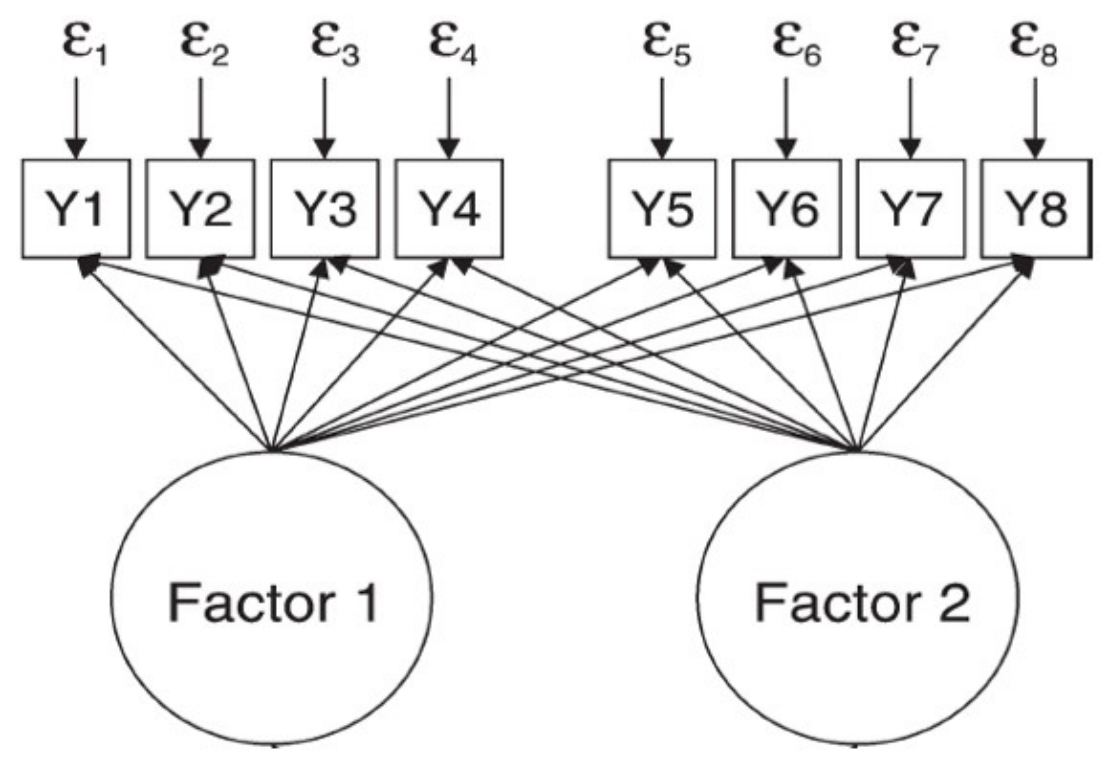

Figura 1: Representación del Análisis Exploratorio

Fuente: Brown [6, p.38]

Brown [6, p.19] indica que: en el AFC, un investigador debe especificar por adelantado varios aspectos clave del modelo de factores (por ejemplo, número de factores, patrones de carga de indicadores y factores). 


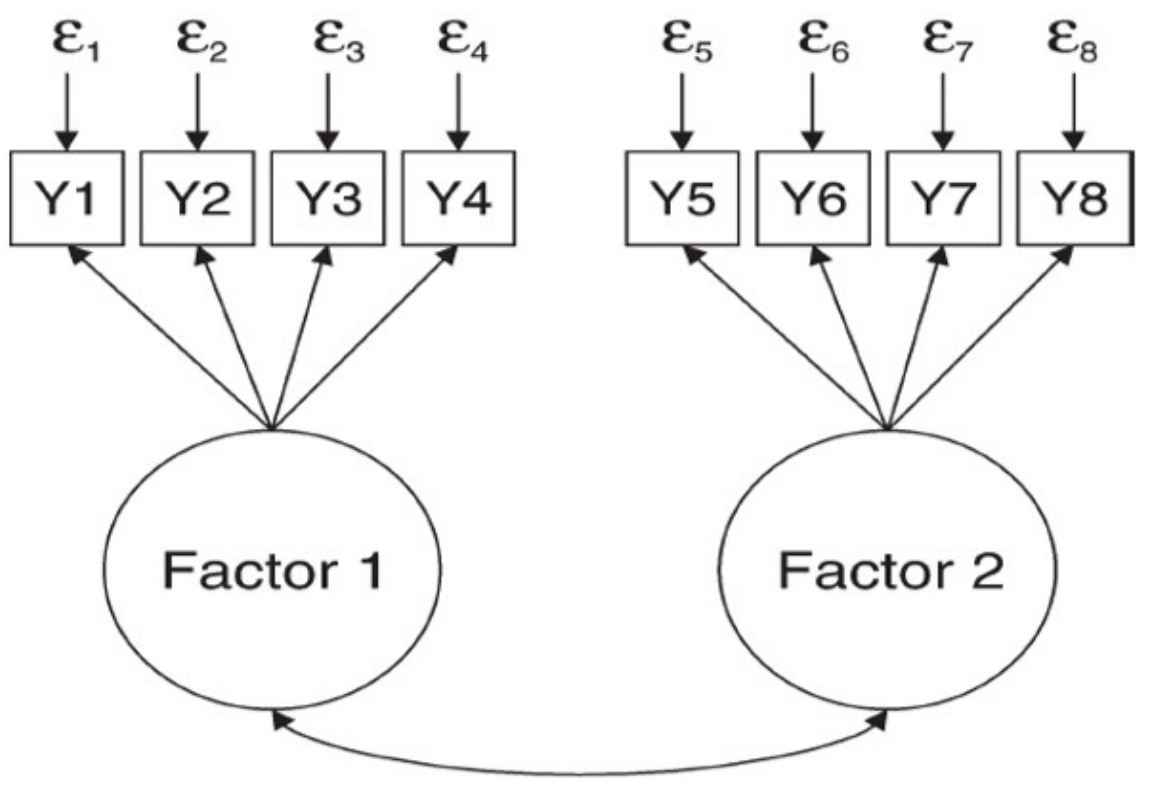

Figura 2: Representación del Análisis Confirmatorio Fuente: Brown [6, p.38]

\section{Análisis Factorial Confirmatorio}

El análisis factorial confirmatorio es una técnica de análisis multivariante, la cual parte de un gran número de variables de las cuales determinados conjuntos de estas variables explican de manera cuantitativa a nuevas variables denominadas factores, es decir, de manera particular un conjunto de variables que están correlacionadas entre sí generan una dimensión que representará a estas variables que se conoce como factor, así de manera paralela se está confirmando un contenido teórico, a su vez si se tiene conocimiento previo que existen determinados factores que miden una variable compleja la cual se está investigando esta puede ser confirmada con el análisis factorial confirmatorio.

Brown [6, p.1, 35] afirma que: El análisis factorial confirmatorio (AFC) es un tipo de modelo de ecuación estructural que se ocupa específicamente de los modelos de medición, es decir, las relaciones entre las medidas o indicadores observados (por ejemplo, elementos de prueba, puntajes de una prueba, calificaciones de observación de comportamiento) y variables o factores latentes. El AFC requiere una sólida base empírica o conceptual para guiar la especificación y evaluación del modelo factorial. En consecuencia, el AFC se usa típicamente en fases posteriores del desarrollo de escala o la validación de construcciones, después de que la estructura subyacente se haya establecido provisionalmente mediante análisis empíricos previos utilizando AFE, así como sobre bases teóricas.

Jöreskog et al. [10, p.283] afirma que: Un análisis factorial confirmatorio comienza definiendo las variables latentes que uno quisiera medir. Esto se basa en una teoría sustantiva y/o conocimiento previo. Luego se selecciona las variables observables para medir las variables latentes. Esta construcción debe seguir ciertas reglas de correspondencia. 


\subsection{Modelo de Análisis Factorial Confirmatorio}

Según Jöreskog et al. 10, el modelo factorial general es representado como:

$$
X=\tau_{x}+\Lambda_{x} \xi+\delta
$$

donde: $\mathrm{X}$ es la variable aleatoria observable de orden $\mathrm{p} \times 1 ; \tau_{x}$ es el vector de constantes de orden $\mathrm{p} \times 1 ; \Lambda_{x}$ es la matriz de cargas Factoriales de orden $\mathrm{p} \times \mathrm{q} ; \xi$ es el vector aleatorio independiente de orden $\mathrm{q} \times 1$, con $E(\xi)=\kappa$ y $V(\xi)=\Phi ; \kappa$ es el vector de medias esperadas de $\xi$ de orden $\mathrm{q} \times 1 ; \Phi$ es la covarianza de factores o matriz de correlación de orden q $\times \mathrm{q}$; $\delta$ es el vector aleatorio independiente de orden $\mathrm{p} \times 1$, con $V(\delta)=\Theta_{\delta}$; $\Theta_{\delta}$ es la matriz de covarianzas de los errores, de orden $\mathrm{p} \times \mathrm{p}$; $\mathrm{p}$ es el número de variables observables o ítems; q es el número de factores o variables latentes.

En la ecuación (1), se debe considerar las siguientes condiciones: $E(\delta)=0$ y $\operatorname{Cov}\left(\xi \delta^{\prime}\right)=0$, es decir, las variables latentes y los errores son incorrelacionados.

Además de la ecuación (1), el vector de parámetros a estimar estaría definido como $\theta=$ $\left(\tau_{x}, \Lambda_{x}, \kappa, \Phi, \Theta_{\delta}\right)$, es decir, se buscaría estimar 5 elementos (entre vectores y matrices) del modelo; es por esto último, que se prefiere reducir el número de elementos considerando $\kappa=0$, quedando la ecuación (1) expresada como:

$$
X=\Lambda_{x} \xi+\delta
$$

De la ecuación (2), las condiciones a considerar serían $E(\xi)=0, E(\delta)=0$ y $\operatorname{Cov}\left(\xi \delta^{\prime}\right)=0$ (incorrelación entre las variables latentes y los errores). Por lo que, el vector de parámetros a estimar sería $\theta=\left(\Lambda_{x}, \Phi, \Theta_{\delta}\right)$.

Para definir la estructura del Análisis Factorial Confirmatorio, denominada también secuencia de modelización del AFC, se procedió a revisar investigaciones de diferentes autores como Long [12, Bollen [5], Mueller [15], Batista et al. [4], Brown [6], entre otros, por lo cual se procederá a considerar la siguiente secuencia: especificación del modelo, identificación del modelo, estimación de los parámetros del modelo, evaluación y/o adecuación del modelo, y la reespecificación del modelo.

\subsubsection{Especificación del Modelo}

En esta fase el investigador debe realizar una revisión exhaustiva de la variable o variables de investigación que intervienen en el modelo del análisis factorial confirmatorio, tanto como sus subvariables es decir sus dimensiones y los indicadores que corresponden a los mismos. A partir de las revisiones teóricas se procede a la formalización matemática, representado a partir de un diagrama de relaciones causales (Path diagrama).

\subsubsection{Identificación del Modelo}

La identificación del modelo corresponde básicamente a verificar si la representación del modelo de ecuaciones estructurales cumple mínimamente con los requerimientos básicos a toda ecuación, cuando esta observada desde el punto de vista de la solución, considerando que en el camino de esta no necesariamente se tendrá una solución única puesto que puede haber muchos caminos, es decir muchas soluciones y en caso contrario a las anteriores no tener solución. 


\subsubsection{Estimación de los parámetros del Modelo}

En la actualidad existen muchos métodos para la estimación de los parámetros debido al desarrollo de los programas computacionales, como son:

- Método de Máxima Verosimilitud (ML).

- Método de Mínimos Cuadrados Ponderados o de Libre Distribución Asintótica (WLS / $\mathrm{ADF})$.

- Método de Mínimos Cuadrados No Ponderados (ULS).

- Método de Mínimos Cuadrados Generalizados (GLS).

- Método de Mínimos Cuadrados de Libre Escala o Ponderados Diagonalmente (SLS / DWLS).

Para la estimación del modelo, se parte de la función de ajuste, la cual según Jöreskog et al. [10, p.508], se representa como:

$$
F(s ; \sigma(\theta))=[s-\sigma(\theta)]^{\prime} V[s-\sigma(\theta)]
$$

donde: $s=\left(s_{11}, s_{21}, s_{22}, s_{31}, \ldots, s_{p p}\right)$ es un vector de la Matriz de Varianza y Covarianza muestral de orden $\mathrm{p}(\mathrm{p}+1) / 2 \times 1 ; \sigma(\theta)=\left(\sigma_{11}, \sigma_{21}, \sigma_{22}, \sigma_{31}, \ldots, \sigma_{p p}\right)$ es un vector de la Matriz de Varianzas y Covarianzas poblacional de orden $\mathrm{p}(\mathrm{p}+1) / 2 \times 1$; p es el total de variables observables; $\mathrm{V}$ es una matriz definida positiva fija o una matriz aleatoria que converge en probabilidad a una matriz definida positiva $\bar{V}$.

- Método de Máxima Verosimilitud (ML): Bollen [5] presenta a la función de ajuste por este método de estimación partiendo de la multinormalidad de los datos, como:

$$
F_{M L}(S ; \hat{\Sigma})=\operatorname{tr}\left(S \hat{\Sigma}^{-1}\right)-\ln \left|S \hat{\Sigma}^{-1}\right|-p
$$

- Método de Mínimos Cuadrados Ponderados (WLS): Es utilizado cuando las variables no presentan distribución normal, el cual es respaldado por Jöreskog et al. [10, pp.508-509], cuya función de ajuste es:

$$
F_{W L S}(s ; \sigma(\theta))=[s-\sigma(\theta)]^{\prime} W^{-1}[s-\sigma(\theta)]
$$

- Método de Mínimos Cuadrados No Ponderados (ULS): Según Harman y citado por Jöreskog et al. [10, pp. 260 - 261], menciona que la función de ajuste es:

$$
F_{U L S}(S ; \hat{\Sigma})=(1 / 2) \operatorname{tr}\left[(S-\hat{\Sigma})^{2}\right]
$$

- Método de Mínimos Cuadrados Generalizados (GLS): Jöreskog y Goldberger [9, p.244], fundamentan que la función GLS es de libre escala, cuya función de ajuste es:

$$
F_{G L S}(S ; \hat{\Sigma})=(1 / 2) \operatorname{tr}\left(\left[(S-\hat{\Sigma}) S^{-1}\right]^{2}\right)
$$

- Método de Mínimos Cuadrados de Libre Escala (SLS): Es empleado cuando no se cumple el supuesto de normalidad en las variables debido a que las variables son de escala ordinal. Según Arbuckle [3, p.585], la función de ajuste es:

$$
F_{S L S}(S ; \hat{\Sigma})=(1 / 2) \operatorname{tr}\left(\left[(S-\hat{\Sigma}) \operatorname{Diag}(S)^{-1}\right]^{2}\right)
$$




\subsubsection{Evaluación y/o adecuación del Modelo}

En esta fase el investigador deberá evaluar si el modelo propuesto se ajusta a los datos. Ya que pueden darse situaciones en la que existan varianzas negativas lo cual se sabe no tienen sentido y mostraría evidencia de cálculos mecánicos pero que carecen de coherencia matemática. Se reviso los libros de los autores: Bollen [5], Mueller [15], Catena et al. [7, Uriel y Aldas [19], Hair et al. [8], Brown [6] y Jöreskog et al. [10]; asimismo, los artículos de Batista et al [4], Morales et al. [14]; resumiendo los indicadores de bondad de ajuste y sus respectivos valores referenciales en la siguiente tabla::

Tabla 1: Índices de Bondad de Ajuste

\begin{tabular}{|c|c|}
\hline Medidas & Índice \\
\hline \multirow{6}{*}{$\begin{array}{l}\text { Bondad de } \\
\text { Ajuste } \\
\text { Absoluto }\end{array}$} & Chi Cuadrado, $(p>\alpha)$ \\
\hline & GFI $\geq 0.90$ \\
\hline & $\mathrm{RMSR}(\mathrm{RMR}) \leq 0.08$ \\
\hline & RMSEA $\leq 0.08$ \\
\hline & NCP pequeño \\
\hline & SNCP pequeño \\
\hline \multirow{8}{*}{$\begin{array}{l}\text { Bondad de } \\
\text { Ajuste } \\
\text { Incremental }\end{array}$} & AGFI $\geq 0.90$ \\
\hline & RGFI $\geq 0.90$ \\
\hline & NFI $\geq 0.90$ \\
\hline & $\mathrm{CFI} \geq 0.90$ \\
\hline & IFI $\geq 0.90$ \\
\hline & RFI $\geq 0.90$ \\
\hline & $\mathrm{TLI}=\mathrm{NNFI} \geq 0.90$ \\
\hline & MFI $\geq 0.90$ \\
\hline \multirow{5}{*}{$\begin{array}{l}\text { Bondad de } \\
\text { Ajuste de } \\
\text { Parsimonía }\end{array}$} & AIC pequeño \\
\hline & CAIC pequeño \\
\hline & PGFI $\geq 0.90$ \\
\hline & BCC pequeño \\
\hline & BIC pequeño \\
\hline
\end{tabular}

Fuente: Elaboración Propia.

\subsubsection{Reespecificación del Modelo}

Esta fase se realiza sólo en caso de ser necesaria, cuando se encuentre alguna inconsistencia en la fase anterior, como obtener estimaciones con signos contrarios a los esperados de manera teórica. Si fuera necesario realizar alguna modificación en la estructura del modelo, es importante conocer los aspectos teóricos de tal manera que no se caiga en contradicciones. 


\section{Resultados}

A continuación, se describen algunas características personales y laborales, encontrándose que, del total de 242 docentes evaluados, el $20.7 \%$ son de sexo femenino siendo este el grupo menos representativo. Respecto al estado civil de los docentes el $57.4 \%$ son solteros y el $31.8 \%$ son casados. El $55.4 \%$ de los docentes no tienen hijos, el $27.7 \%$ tiene de uno a dos hijos, el $12.8 \%$ tienen de 3 a más hijos. Es importante mencionar que el $31.0 \%$ de los docentes tienen un tiempo de trabajo como máximo un año, el $43.8 \%$ tienen de uno a cuatro años, el $12.8 \%$ tienen de 5 a 10 años de antigüedad y el $8.7 \%$ tienen más de 10 años de antigüedad. Por otro lado, el $28.1 \%$ de los docentes tienen contrato por tiempo definido con la institución, el $15 \%$ son docentes que tienen una condición laboral estable, y el resto de los docentes tienen otra condición laboral (contratos con tiempo indefinido, entre otros).

El modelo de dos factores de la escala de satisfacción laboral se representa en la figura 3 , y el modelo de un factor en la figura 4. Asimismo, en la tabla 2 y tabla 3 se presentan los valores estimados y los indicadores de bondad de ajuste de cada modelo.

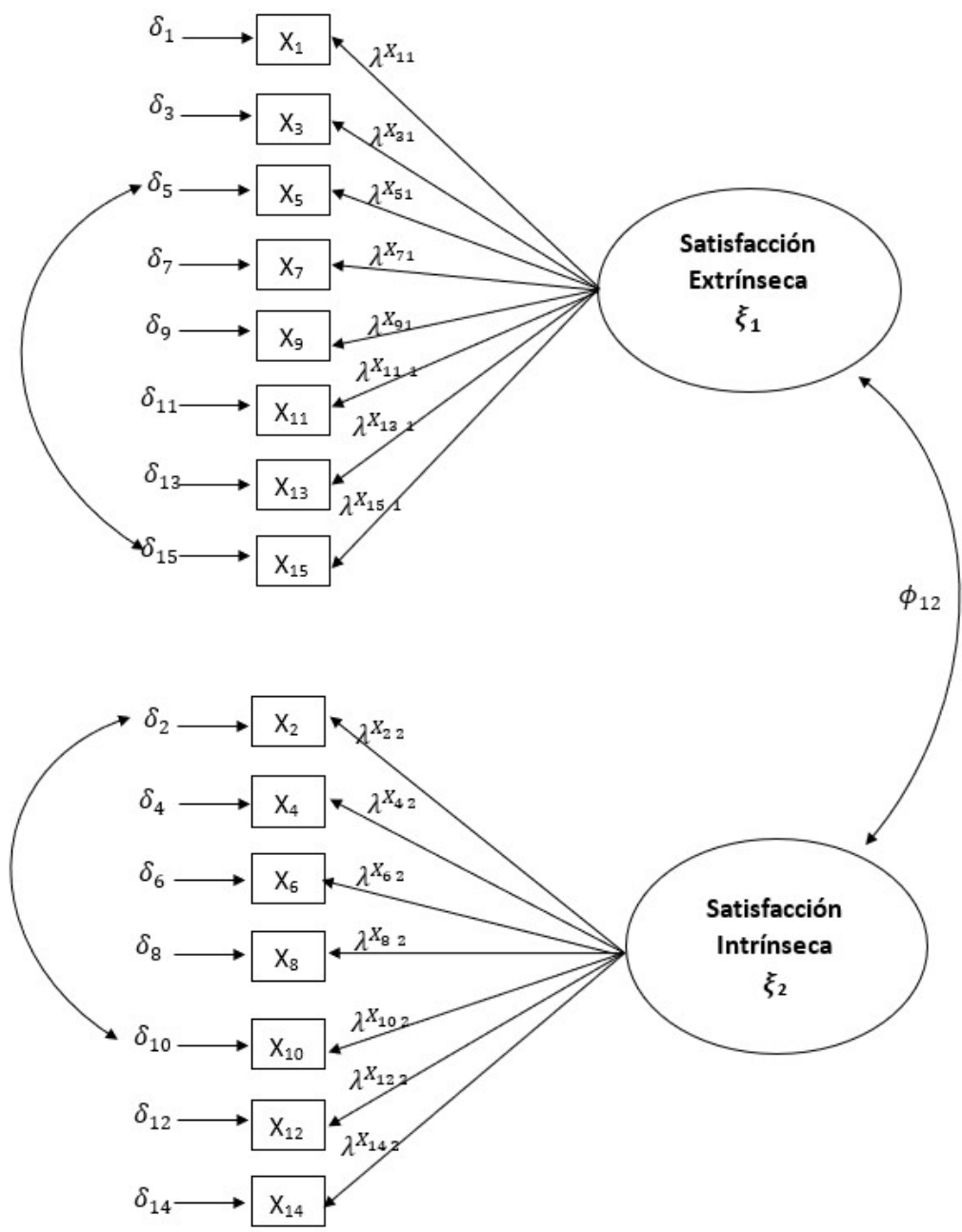

Figura 3: Path Diagrama del modelo de medida de dos factores Fuente: Elaboración Propia. 


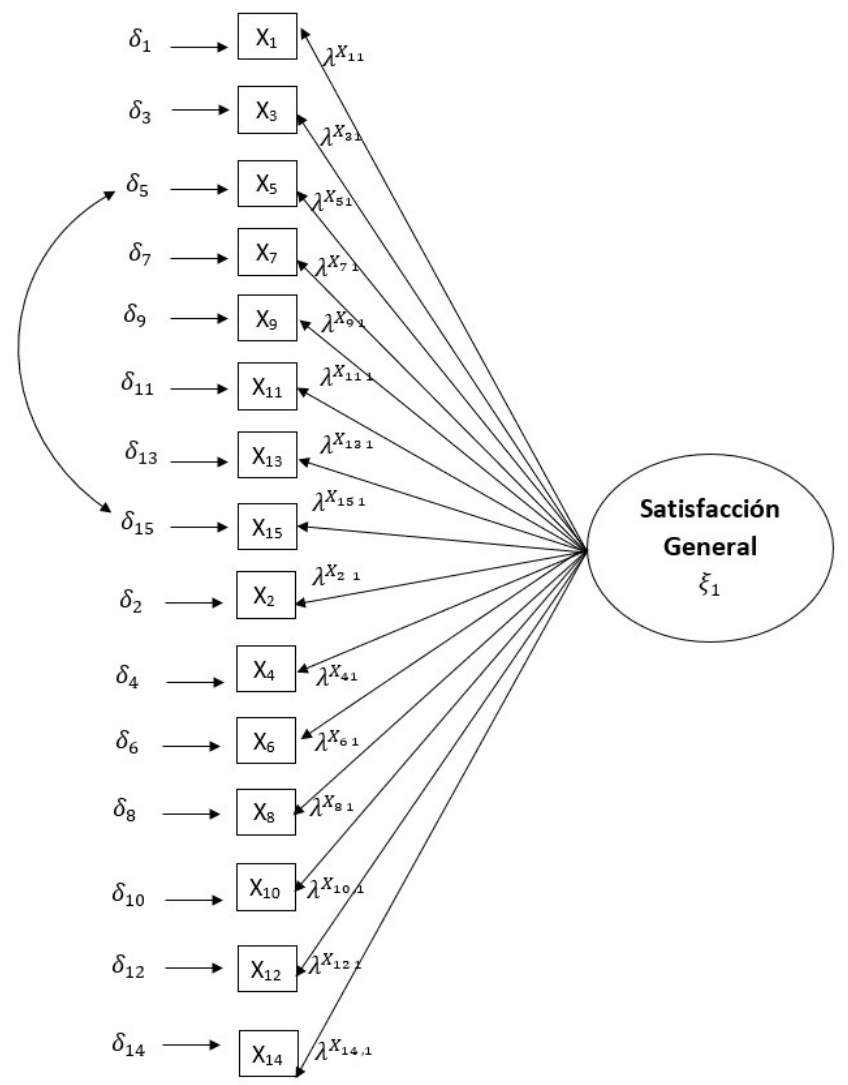

Figura 4: Path Diagrama del modelo de medida de un factor Fuente: Elaboración Propia.

Tabla 2: Parámetros estimados

\begin{tabular}{|c|c|c|c|c|c|c|}
\hline & \multicolumn{3}{|c|}{ Dos Factores } & \multicolumn{3}{c|}{ Un Factor } \\
\hline Ítem & $\lambda_{i j}$ & $\lambda_{i j}^{2}$ & $e_{i}^{2}$ & $\lambda_{i j}$ & $\lambda_{i j}^{2}$ & $e_{i}^{2}$ \\
\hline \hline 1 & 0.6720 & 0.4516 & 0.5484 & 0.6640 & 0.4409 & 0.5591 \\
\hline 2 & 0.5200 & 0.2704 & 0.7296 & 0.4940 & 0.2440 & 0.7560 \\
\hline 3 & 0.3660 & 0.1340 & 0.8660 & 0.3630 & 0.1318 & 0.8682 \\
\hline 4 & 0.6640 & 0.4409 & 0.5591 & 0.6520 & 0.4251 & 0.5749 \\
\hline 5 & 0.7250 & 0.5256 & 0.4744 & 0.7160 & 0.5127 & 0.4873 \\
\hline 6 & 0.6010 & 0.3612 & 0.6388 & 0.5910 & 0.3493 & 0.6507 \\
\hline 7 & 0.5970 & 0.3564 & 0.6436 & 0.5920 & 0.3505 & 0.6495 \\
\hline 8 & 0.5930 & 0.3516 & 0.6484 & 0.5840 & 0.3411 & 0.6589 \\
\hline 9 & 0.7940 & 0.6304 & 0.3696 & 0.7840 & 0.6147 & 0.3853 \\
\hline 10 & 0.7710 & 0.5944 & 0.4056 & 0.7380 & 0.5446 & 0.4554 \\
\hline 11 & 0.7270 & 0.5285 & 0.4715 & 0.7180 & 0.5155 & 0.4845 \\
\hline 12 & 0.7180 & 0.5155 & 0.4845 & 0.7060 & 0.4984 & 0.5016 \\
\hline 13 & 0.6530 & 0.4264 & 0.5736 & 0.6480 & 0.4199 & 0.5801 \\
\hline 14 & 0.6080 & 0.3697 & 0.6303 & 0.5980 & 0.3576 & 0.6424 \\
\hline 15 & 0.5750 & 0.3306 & 0.6694 & 0.5670 & 0.3215 & 0.6785 \\
\hline
\end{tabular}

Fuente: Elaboración Propia.

Para evaluar la confiabilidad se obtuvo el coeficiente Alfa de Cronbach, encontrándose un valor de 0.867 (86.7\%, IC (95\%): $83.2 \%$ - 89.7\%), es decir, una alta confiabilidad, esto es, la escala de satisfacción de Warr, Cox y Wall tiene una alta confiabilidad en la cual puede 
Tabla 3: Índices de Bondad de Ajuste

\begin{tabular}{|c|c|c|c|c|c|c|c|}
\hline Modelo & $\chi^{2}$ & gl & GFI & RMSR & AGFI & NFI & RFI \\
\hline \hline Dos factores & 59.024 & 87 & 0.984 & 0.066 & 0.978 & 0.969 & 0.963 \\
\hline Un factor & 63.185 & 89 & 0.983 & 0.070 & 0.977 & 0.967 & 0.961 \\
\hline
\end{tabular}

Fuente: Elaboración Propia.

denominarse que existe una muy buena consistencia interna de la escala. En la tabla 4, se observa que los coeficientes de correlación total si el ítem se elimina están por encima de 0.30, lo cual indica que si se retira alguna de las variables observables este proceso no contribuirá a ganar un mayor nivel de confiabilidad.

Tabla 4: Estadísticas si el ítem se elimina

\begin{tabular}{|c|c|c|c|c|}
\hline Ítem & Media & Varianza & Correlación & Alfa de Cronbach \\
\hline P1 & 52.89 & 102.362 & 0.460 & 0.861 \\
\hline P2 & 52.24 & 105.650 & 0.433 & 0.862 \\
\hline P3 & 52.04 & 108.667 & 0.406 & 0.863 \\
\hline P4 & 52.91 & 101.751 & 0.548 & 0.856 \\
\hline P5 & 52.56 & 102.198 & 0.535 & 0.857 \\
\hline P6 & 52.45 & 103.029 & 0.523 & 0.858 \\
\hline P7 & 53.67 & 102.870 & 0.520 & 0.858 \\
\hline P8 & 52.35 & 104.394 & 0.520 & 0.858 \\
\hline P9 & 52.59 & 100.210 & 0.642 & 0.852 \\
\hline P10 & 53.40 & 100.440 & 0.506 & 0.859 \\
\hline P11 & 53.05 & 101.305 & 0.574 & 0.855 \\
\hline P12 & 53.14 & 101.239 & 0.593 & 0.854 \\
\hline P13 & 52.40 & 103.620 & 0.571 & 0.856 \\
\hline P14 & 52.52 & 104.309 & 0.490 & 0.859 \\
\hline P15 & 53.26 & 103.586 & 0.387 & 0.866 \\
\hline
\end{tabular}

Fuente: Elaboración Propia.

Tabla 5: Coeficientes de fiabilidad robustos

\begin{tabular}{|l|c|c|c|c|c|}
\hline \multicolumn{1}{|c|}{ Coeficientes } & Fiabilidad & $\begin{array}{c}\text { Satisfacción } \\
\text { General de } \\
\text { 1 Factor }\end{array}$ & $\begin{array}{c}\text { Satisfacción } \\
\text { General de } \\
\text { 2 Factores }\end{array}$ & $\begin{array}{c}\text { Satisfacción } \\
\text { Extrínseca }\end{array}$ & $\begin{array}{c}\text { Satisfacción } \\
\text { Intrínseca }\end{array}$ \\
\hline \multirow{2}{*}{ Estimados } & $\alpha_{\text {ordinal }}$ & 0.9067 & 0.9116 & 0.8452 & 0.8281 \\
\cline { 2 - 6 } & Compuesta & 0.9085 & 0.9134 & 0.8497 & 0.8302 \\
\hline \multirow{2}{*}{ Estandarizados } & $\alpha_{\text {ordinal }}$ & 0.8456 & 0.8524 & 0.7543 & 0.7293 \\
\cline { 2 - 6 } & Compuesta & 0.8467 & 0.8535 & 0.7575 & 0.7300 \\
\hline
\end{tabular}

Fuente: Elaboración Propia.

En la tabla 5 se obtuvieron medidas de fiabilidad robustas cuyos valores superaron 0.80 , como el coeficiente de confiabilidad compuesto $\omega=0.9085$ (90.85\%) y en el modelo bifactorial $\omega=$ 0.9134(91.34\%). En general, los diferentes coeficientes de fiabilidad para el modelo unifactorial como bifactorial a partir de los parámetros estimados estandarizados como no estandarizados para la Satisfacción General son superiores a 0.80, es decir, la consistencia interna de la escala se encuentra en un nivel muy bueno. 


\section{Discusión}

En lo que respecta a la Satisfacción Laboral, y en particular el cuestionario de "Satisfacción Laboral de Warr, Cox y Wall" para los diferentes grupos evaluados en múltiples investigaciones, se ha comprobado su validez las cuales han sido sustentadas de manera teórica y confirmadas a través de análisis cuantitativos. Sin embargo, si se desea profundizar respecto a su estructura dimensional, es decir si es unidimensional o bidimensional, se debe mantener especial prudencia. Las investigaciones presentadas por Frederick Irving Herzberg a fines de los años 60 muestran evidencia que la Satisfacción Laboral, tiene dos componentes importantes conocidos como Motivación e Higiene, según la "Teoría de los dos factores", Manso [13, Pardee [16] y Untref [18]. Adicionalmente, la Satisfacción Laboral es un estado general, es decir, "único", tal como mencionan Andresen et al. [2, p.719] y Locke [11].

El cuestionario de Satisfacción Laboral de Warr, Cox y Wall, fue construido en base a las teorías mencionadas, en donde se mencionan a la Satisfacción Laboral General, la Satisfacción intrínseca y la Satisfacción extrínseca, las cuales se presentan en los modelos de medidas (Figura 3 y Figura (4), obteniéndose un buen ajuste bajo la concepción del modelo de medida unidimensional que estaría siendo representado por la Satisfacción Laboral General como también el modelo de medida bidimensional en el que se consideraron sus dos dimensiones (satisfacción extrínseca e intrínseca). En resumen, el hecho de tener teorías que argumenten la bidimensionalidad de la Satisfacción Laboral, no la excluyen de ser unidimensional, tal como se aprecia en la presente investigación.

\section{Conclusión}

A partir del proceso de validación realizado, se concluye que, la estructura interna del cuestionario de "Satisfacción Laboral de Warr, Cox y Wall" para docentes de educación superior es válida. Mediante el método de estimación de Mínimos Cuadrados de Libre Escala para un modelo de medida unifactorial como para un modelo de medida bifactorial, en las cuales se guardó la coherencia basada en los fundamentos teóricos, se obtuvieron estadísticos Chi Cuadrado (discrepancia) 59.024 y 63.185 respectivamente, pequeños en comparación con otras estimaciones realizadas, para el modelo unifactorial GFI $=0.984 \geq 0.90, \mathrm{AGFI}=0.978 \geq 0.90, \mathrm{NFI}=0.969$ $\geq 0.90, \mathrm{RFI}=0.963 \geq 0.90, \mathrm{RMSR}=0.066 \leq 0.08 ; \mathrm{y}$ en el modelo bifactorial GFI $=0.983 \geq$ $0.90, \mathrm{AGFI}=0.977 \geq 0.90, \mathrm{NFI}=0.967 \geq 0.90, \mathrm{RFI}=0.961 \geq 0.90, \mathrm{RMSR}=0.070 \leq 0.08$, comprobándose que las medidas se encuentran dentro de los parámetros adecuados. (De manera referencial se hace presente que al realizar el Método de Estimación de Máxima Verosimilitud el estadístico Chi Cuadrado es 112.556 con un p valor de 0.034 siendo este superior al nivel de significación del $1 \%$ obteniendo medidas de bondad de ajustes adecuadas. Es importante mencionar que, se ha encontrado el mismo patrón y sus parámetros estimados son significativos).

En segundo lugar, se concluye que el cuestionario de "Satisfacción Laboral de Warr, Cox y Wall" para docentes de educación superior es fiable. A partir del análisis de fiabilidad realizado de manera global para la satisfacción general se obtuvo un Alfa de Cronbach de 0.867, es decir con un nivel de confiabilidad alto, adicionalmente se realizó esto mismo para la satisfacción extrínseca como intrínseca obteniéndose también niveles altos de confiabilidad. Se considero trabajar adicionalmente con medidas de confiabilidad robustas como el coeficiente de fiabilidad compuesta y el coeficiente de fiabilidad alfa ordinal, realizando los cálculos basados en sus cargas factoriales obtenidas a partir del análisis factorial confirmatorio de los diferentes modelos de medida basados en la satisfacción general como para la satisfacción intrínseca como extrínseca obteniéndose niveles de confiabilidad, adecuados a altos. (Tabla 5) 
En esta investigación se ha presentado evidencias de validez y confiabilidad de la Escala de Satisfacción Laboral de Warr, Cox y Wall en su estructura interna, obteniendo como producto final un test, el cual presenta evidencia de que su uso e interpretación son correctos para medir la Satisfacción Laboral en docentes de educación superior.

Finalmente se puede afirmar que la escala de "Satisfacción Laboral de Warr, Cox y Wall"para docentes de educación superior es válido en su estructura interna y fiable.

\section{Referencias bibliográficas}

[1] Albright, J. (2008). Confirmatory Factor Analysis using Amos, LISREL, and Mplus. The Trustees of Indiana University. https://www.researchgate.net/profile/Roger_Keller_ Celeste/post/For_the_SF_36_questionnaire_which_is_the_best_way_to_analyze_and_show_the _results/attachment/59d624bbc49f478072e99f51/AS \%3A272152517906442\%401441897625 006/download/cfa+comparison+lisrel+amos+mplus.pdf

[2] Andresen, M., Domsch, M. y Cascorbi, A. (2007). Working Unusual Hours and Its Relationship to Job Satisfaction: A Study of European Maritime Pilots. Journal of Labor Research, 28(4), 714-734. https://doi.org/10.1007/s12122-007-9010-5

[3] Arbuckle, J. (2012). IBM SPSS Amos 21 - User's Guide. IBM SPSS. https://usermanual. wiki/Ibm/IbmIbmLaptopSpssAmos21UsersManual431345.461810123/view

[4] Batista-Fogueta, J., Coenders, G., Alonso, J. (2004). Análisis factorial confirmatorio. $\mathrm{Su}$ utilidad en la validación de cuestionarios relacionados con la salud. Medicina Clínica, 122(1), 21-27. https://www.elsevier.es/es-revista-medicina-clinica-2-articulo-analisisfactorial-confirmatorio-su-utilidad-13057542

[5] Bollen, K. (1989). Structural Equations with Latent Variables. Wiley.

[6] Brown, T. (2015). Confirmatory Factor Analysis for Applied Research.. (2 $2^{a}$ ed.). The Guilford Press.

[7] Catena, A., Ramos, M., Trujillo, H. (2003). Análisis Multivariado. Un Manual para Investigadores. Editorial Biblioteca Nueva.

[8] Hair, J., Anderson, R., Black, W., Babin, B. (2014). Multivariate Data Analysis. (7 ${ }^{a}$ ed.). Pearson New International Edition.

[9] Jöreskog, K., Golberger, A. (1972). Factor Analysis by Generalized Least Squares. Psychometrika, 37(3), 243-260. https://www.researchgate.net/profile/Karl_Joereskog/publication /24062019_Factor_Analysis_by_Generalized_Least_Squares/links/0046352b8b0791133d0000 00/Factor-Analysis-by-Generalized-Least-Squares.pdf

[10] Jöreskog, K., Olsson, U., Wallentin, F. (2016). Multivariate Analysis with Lisrel. Springer.

[11] Locke, E. (1976). The nature and causes of job satisfaction. Handbook of industrial and organizational psychology. ( $1^{a}$ ed.), 1297-1349. Rand - Mc Nally College.

[12] Long, J. (1983). Confirmatory Factor Analysis: A Preface to Lisrel. Sage Publications Inc.

[13] Manso, J. (2002). El Legado de Frederick Irving Herzberg. Revista Universidad EAFIT, 128(4), 79-86. https://www.researchgate.net/publication/26422979_El_legado_de_Frederick_ Irving_Herzberg 
[14] Morales, V., Hernández, A., Blanco, A. (2005). Evaluación de la Calidad en los programas de actividad física. Psicothema, 17(2), 311-317. http://www.psicothema.com/psicothema. asp?id=3105

[15] Mueller, R. (2005). Analyse Fonctionnelle - Basic Principles of Structural Equation Modeling. An Introduction to Lisrel and EQS. Springer.

[16] Pardee, R. (1990). Analyse Fonctionnelle - Motivation Theories of Maslow, Herzberg, McGregor 85 McClelland. A Literature Review of Selected Theories Dealing with Job Satisfaction and Motivation. Educational Resources Information Center. https://eric.ed.gov/?id=ED316767

[17] Pérez, J., Fidalgo, M. (2013). NTP 394: Satisfacción Laboral: Escala general de Satisfacción. Centro Nacional de Condiciones de Trabajo. https://saludlaboralydiscapacidad.org/wpcontent/uploads/2019/05/NTP-394-Satisfacci \%C3\%B3n-laboral-escala-general-de-satisfa cci $\%$ C3\%B3n.pdf

[18] Herzberg, F. (2011). 10 Teoría de la Motivación - Higiene. Fundamentos de Estrategia Organizacional. (Trad. UNTREF), Universidad Nacional de Tres de Febrero Virtual. (Trabajo original publicado en 1954, Work of the Nature of Man. Cleveland: The World of Publishing Company). http://materiales.untrefvirtual.edu.ar/documentos_extras/ 1075_Fundamentos_de_estrategia_organizacional/10_Teoria_de_la_organizacion.pdf

[19] Uriel, E., Aldás, J. (2005). Análisis Multivariante Aplicado. (1 ${ }^{a}$ ed.). Thomson/ Paraninfo. 